SNUTP-96-81

\title{
Chiral Corrections to the Axial Charges of the Octet Baryons from Quenched QCD
}

\author{
Myunggyu Kim and Seyong Kim \\ Center for Theoretical Physics, Seoul National University, Seoul 151-742, Korea \\ skim@ctp.snu.ac.kr \\ mgkim@galatica.snu.ac.kr
}

\begin{abstract}
We calculate one-loop correction to the axial charges of the octet baryons using quenched chiral perturbation theory, in order to understand chiral behavior of the axial charges in quenched approximation to quantum chromodynamics (QCD). In contrast to regular behavior of the full QCD chiral perturbation theory result, $c_{0}+c_{l 2} m_{\pi}^{2} \ln m_{\pi}^{2}+\cdots$, we find that the quenched chiral perturbation theory result, $c_{0}^{Q}+\left(c_{l 0}^{Q}+c_{l 2}^{Q} m_{\pi}^{2}\right) \ln m_{\pi}^{2}+c_{2}^{Q} m_{\pi}^{2}+\cdots$, is singular in the chiral limit.
\end{abstract}


Lattice quantum chromodynamics (QCD) simulation allows us to investigate low energy phenomena of the strong interaction using first principles of quantum field theory. It is well suited for understanding non-perturbative nature of the strong interaction. Thus far, this method has been successfully employed in calculating various low energy QCD related quantities [1].

However, many of physical observables calculated in lattice QCD use so-called "quenched approximation" in which vacuum polarization effects coming from quark-antiquark pair creation annihilation is neglected. This approximation is used due to the extensive computational cost in lattice simulation of full QCD, in particular, of light quark system. Such truncation, otherwise in a first principle calculation, can cause undesirable effects on what we are interested in. It is often difficult to estimate such effects quantitatively. In addition to this, most cases of the current lattice simulations are done with bare quark masses which produce pion masses heavier than experimental value. Extrapolation of heavier mass results from simulations to experimental pion mass region is necessary. Based on the observation that one may use Taylor expansion for the mass dependence of physical quantities when the mass is small, a linear or quadratic fit has been used for the chiral extrapolation. One may question wisdom of such an extrapolation under the quenched approximation.

In order to understand quenching effects on chiral limit of various physical observables in light meson system, recently, Sharpe devised a rule + digrammatic method [2]. Bernard and Goltermann [3] developed a systematic way based on the symmetry of quenched QCD (QQCD). The latter method is in similar spirit to chiral perturbation theory and an extension of the same idea to baryonic system is constructed by Labrenz and Sharpe [4]. Using their method, they found that the chiral behaviors of many physical quantities in the QQCD are different from those in the full QCD and there are indeed sickness caused by quenched approximation. The question is whether such differences are numerically noticeable in physical pion mass region. If at the physical mass region, the deviation is small, we may safely neglect it. Numerical investigations on meson mass (pion) revealed that although there is difference between the chiral behavior of the full QCD and that of the QQCD, the magnitude 
is small at the physical pion mass region [5]. Similar conclusion may be drawn on the nucleon mass [6]. However, here, using the method developed in [4, we suggest that the chiral behavior of the axial charge of the octet baryons in QQCD should depart noticeably from that of full QCD in the physical pion mass region and in bare quark mass region currently used in lattice QCD simulation. In this case, a quenched calculation may not be trusted, let alone a linear extrapolation of it.

In the following, we briefly mention the most important ingredients of the quenched chiral perturbation theory $(\mathrm{Q} \chi \mathrm{PT})$. Details are available in [3, 4] and we follow the notations in [4]. Quenched approximation amounts to neglecting the quark determinant. Cancelling the quark determinant can be achieved by introducing additional bosonic degrees of freedom, $\tilde{q}_{i}$, corresponding to each flavor of the quark field $q_{i}$. Each of $\tilde{q}_{i}$ has the same mass, charge etc as the original quark. Since new bosonic degrees of freedom has the same quantum number as fermion degrees of freedom (quarks) except the spin, Gaussian integral over the new degrees of freedom matches exactly the determinant from the quark degrees of freedom with opposite power. Due to new bosonic degrees of freedom, then, the symmetry of QQCD system becomes the graded symmetry $U(3 \mid 3) \times U(3 \mid 3)$ for the quenched system of light quarks, $(\mathrm{u}, \mathrm{d}, \mathrm{s})$. This symmetry determines the form of the interactions among the pseudoscalar mesons in $\mathrm{Q} \chi \mathrm{PT}$.

The dynamics of the mesons is conveniently described by an Hermitian $6 \times 6$ matrix field $\Phi$. A component $\Phi_{i j}$ has the same transformation properties as the operator $Q_{i} \bar{Q}_{j}$ where $Q=(u, d, s, \tilde{u}, \tilde{d}, \tilde{s})$. As in $\chi \mathrm{PT}$ (chiral perturbation theory), the following is useful in constructing the Lagrangian:

$$
\begin{gathered}
\Sigma(x)=e^{2 i \Phi(x) / f}, \xi(x)=e^{i \Phi(x) / f}, \\
A_{\mu}(x)=\frac{i}{2}\left(\xi \partial_{\mu} \xi^{\dagger}-\xi^{\dagger} \partial_{\mu} \xi\right), V_{\mu}(x)=\frac{1}{2}\left(\xi \partial_{\mu} \xi^{\dagger}+\xi^{\dagger} \partial_{\mu} \xi\right) .
\end{gathered}
$$

Under $U(3 \mid 3) \times U(3 \mid 3)$, the meson fields transform as

$$
\Sigma \rightarrow L \Sigma R^{\dagger}, \xi \rightarrow L \xi U^{\dagger}(x)=U(x) \xi R^{\dagger}
$$


The axial anomaly breaks this full chiral symmetry at the classical level down to the semidirect product $[S U(3 \mid 3) \times S U(3 \mid 3)] \otimes U(1)$. The reduction in the symmetry introduces the field $\Phi_{0}=\operatorname{str}(\Phi) / \sqrt{3}$ and allows the quenched chiral Lagrangian to include arbitrary functions of $\Phi_{0}$. The resulting Lagrangian in the mesonic sector is then

$$
\begin{array}{r}
\mathcal{L}_{\phi}^{Q}=\frac{f^{2}}{4}\left[\operatorname{str}\left(\partial_{\mu} \Sigma \partial^{\mu} \Sigma^{\dagger}\right)\right. \\
\left.V_{1}\left(\Phi_{0}\right)+2 \mu \operatorname{str}\left(\xi^{\dagger} m \xi+\xi m \xi^{\dagger}\right) V_{2}\left(\Phi_{0}\right)\right] \\
+\alpha_{\Phi} V_{5}\left(\Phi_{0}\right) \partial_{\mu} \Phi_{0} \partial^{\mu} \Phi_{0}-m_{0}^{2} V_{0}\left(\Phi_{0}\right) \Phi_{0}^{2},
\end{array}
$$

where $s t r$ denotes supertrace [3, 团 and $m=\operatorname{diag}\left(m_{u}, m_{d}, m_{s}, m_{u}, m_{d}, m_{s}\right)$. The potentials are normalized as $V_{i}\left(\Phi_{0}\right)=1+\mathcal{O}\left(\Phi_{0}^{2}\right)$. In our calculations, the higher order terms in the potentials will not be needed. In the full chiral theory, the vertex $i\left(\alpha_{\Phi} p^{2}-m_{0}^{2}\right) / 3$ iterates infinitely in the $\eta^{\prime}$ propagater. Thus the $\eta^{\prime}$ acquires the heavy mass and can be integrated out of the effective theory. In the quenched theory, however, only one insertion of the vertex survives and thus the $\Phi_{0}$ remains light. We must therefore keep the last two terms in $\mathcal{L}_{\Phi}^{q}$. Furthermore the lightness of the $\Phi_{0}$ will introduce two additional parameters in the baryon sector as discused below. The couplings, $\alpha_{\Phi}$ and $m_{0}^{2}$ can change usual power counting rule in $\chi \mathrm{PT}$ if they are large. In loop calculation, actual expansion parameters are $\alpha_{\Phi} / 3$ and $m_{0}^{2} / 3$ [3]. This leads one to expect that $\mathrm{Q} \chi \mathrm{PT}$ power counting holds and we calculate only leading behavior of these coupling.

We now define the fields for baryons. QQCD has $q q \tilde{q}, q \tilde{q} \tilde{q}$, and $\tilde{q} \tilde{q} \tilde{q}$ baryons, additional to the ususl $q q q$ baryons. Spin-1/2 baryons belong to an irreducible representation of $S U(3 \mid 3)_{v}$ with dimension 70. $S U(3)$ decomposes it into an 8 each of $q q q$ 's and $\tilde{q} \tilde{q} \tilde{q}$ 's and a $1+8+8+10$ each of $q q \tilde{q}$ 's and $q \tilde{q} \tilde{q}$ 's. The representation including spin-3/2 baryons is 38 dimensional, which consisits of a 10 of $q q q$ 's, a 10 and an 8 of $q q \tilde{q}$ 's, an 8 and a 1 of $q \tilde{q} \tilde{q}$ 's, and a 1 of $\tilde{q} \tilde{q} \tilde{q}$ 's. We call the baryons of the 70(38) simply "octet" ("decuplet") baryons. In $\mathrm{Q} \chi \mathrm{PT}$, the spin-1/2(3/2) baryons are described by the tensor field $B_{i j k}\left(T_{i j k}^{\mu}\right)$. They are defined to have the same transformation properties as the following operators:

$$
\begin{gathered}
B_{i j k}^{\gamma} \sim\left[q_{i}^{\alpha, a} q_{j}^{\beta, b} q_{k}^{\gamma, c}-q_{i}^{\alpha, a} q_{j}^{\gamma, c} q_{k}^{\beta, b}\right] \varepsilon_{a b c}\left(C \gamma_{5}\right)_{\alpha \beta}, \\
T_{\alpha, i j k}^{\mu} \sim\left[q_{i}^{\alpha, a} q_{j}^{\beta, b} q_{k}^{\gamma, c}+q_{i}^{\beta, b} q_{j}^{\gamma, c} q_{k}^{\alpha, a}+q_{i}^{\gamma, c} q_{j}^{\alpha, a} q_{k}^{\beta, b}\right] \varepsilon_{a b c}\left(C \gamma_{\mu}\right)_{\beta \gamma}
\end{gathered}
$$


where $C=i \gamma_{2} \gamma_{0}$ is the charge conjugation matrix and $a, b$ and $c$ are color indices. Both fields have the same transformation properties, exemplified by

$$
B_{\gamma, i j k} \rightarrow(-)^{i^{\prime}\left(j+j^{\prime}\right)+\left(i^{\prime}+j^{\prime}\right)\left(k+k^{\prime}\right)} U_{i i^{\prime}} U_{j j^{\prime}} U_{k k^{\prime}} B_{\gamma, i^{\prime} j^{\prime} k^{\prime}}
$$

where $U \in S U(3 \mid 3)$. The grading factor stems from the fact that the off-diagonal $3 \times 3$ blocks of $U$ are grassman variables. The notations for the indices are as follows: 1 for the anticommuting variables $(i=1,2,3)$ and 0 for the commuting variables $(i=4,5,6)$. The baryon fields satisfy the symmetry properties

$$
\begin{array}{r}
B_{i j k}=(-)^{j k+1} B_{i k j}, \\
B_{i j k}+(-)^{i j+1} B_{j i k}+(-)^{i j+j k+k i+1} B_{k j i} \\
T_{i j k}=(-)^{i j+1} T_{j i k}=(-)^{j k+1} T_{i k j} .
\end{array}
$$

The Lagrangian in the baryon sector at lowest order can be written in terms of the invariant bilinears of the baryon fields. They include the covariant derivative or the field $A^{\mu}$. The covariant derivative of the field $B_{i j k}$ is

$$
D^{\mu} B_{i j k}=\partial^{\mu} B_{i j k}+\left(V^{\mu}\right)_{i i^{\prime}} B_{i^{\prime} j k}+(-)^{i\left(j+j^{\prime}\right)}\left(V^{\mu}\right)_{j j^{\prime}} B_{i j^{\prime} k}+(-)^{(i+j)\left(k+k^{\prime}\right)}\left(V^{\mu}\right)_{k k^{\prime}} B_{i j k^{\prime}}
$$

where $V^{\mu}$ is a vector current defined by eq. (2). The covariant derivarive of the field $T_{i j k}^{\mu}$ takes the same form. We follow [4]'s notations for the contraction of the flavor indices. They are

$$
\begin{gathered}
\bar{C} \Lambda C^{\prime} \equiv \bar{C}_{k j i} \Lambda C_{i j k}^{\prime}, \\
\bar{C} \Lambda E C^{\prime} \equiv \bar{C}_{k j i} \Lambda E_{i i^{\prime}} C_{i^{\prime} j k}^{\prime}, \\
\bar{C} \Lambda C^{\prime} E \equiv \bar{C}_{k j i} \Lambda E_{k k^{\prime}} C_{i j k^{\prime}}^{\prime}(-)^{(i+j)\left(k+k^{\prime}\right)},
\end{gathered}
$$

where $C$ and $C^{\prime}$ are the baryon fields, $E$ is a matrix field, and $\Lambda$ is an arbitrary Dirac matrix. An example of $E$ is $A^{\mu}$.

The lowest order Lagrangian is, then,

$$
\mathcal{L}^{Q}=\mathcal{L}_{\Phi}^{Q}+\mathcal{L}_{B T \Phi}^{Q}
$$


where $\mathcal{L}_{\Phi}^{Q}$ is given in eq. (4) and

$$
\begin{array}{r}
\mathcal{L}_{B T \Phi}^{Q}=i \bar{B} v \cdot D B+i \bar{T}^{\nu} v \cdot D T_{\nu}+\Delta M \bar{T}^{\nu} T_{\nu} \\
+2 \alpha \bar{B} S_{\mu} B A^{\mu}+2 \beta \bar{B} S_{\mu} A^{\mu} B+2 \gamma \bar{B} S_{\mu} B \operatorname{str}\left(A^{\mu}\right) \\
+2 H \bar{T}^{\nu} S_{\mu} A^{\mu} T_{\nu}-\sqrt{\frac{3}{2}} C\left[\bar{T}^{\nu} A^{\nu} B+\bar{B} A_{\nu} T^{\nu}\right]+2 \gamma^{\prime} \bar{T}^{\nu} S_{\mu} T_{\nu} \operatorname{str}\left(A^{\mu}\right) .
\end{array}
$$

The symbols $v$ and $S^{\mu}$ denote the fixed velocity and the spin operator in the heavy baryon formalism [7]. Various other possible terms such as $\bar{B}_{i j k} S^{\mu} A_{\mu, j j^{\prime}} B_{i j^{\prime} k}(-)^{i\left(j+j^{\prime}\right)}$, etc. are not independent due to the symmetry properties given in eq. (8). In caculating the contributions of decuplet intermediate baryons, we assume that $\Delta m \ll m_{\pi}$ and treat the octet and the decuplet baryons as degenerate.

When the flavor indices are restricted to the range $1-3$, the baryon fields in the quenched theory are explicitely related to ones in the full theory as

$$
\begin{gathered}
\left.B_{i j k}\right|_{R}=\frac{1}{\sqrt{6}}\left(\varepsilon_{i j k^{\prime}} B_{k^{\prime} k}^{f u l l}+\varepsilon_{i k k^{\prime}} B_{k^{\prime} j}^{f u l l}\right), \\
\left.B_{i j k}\right|_{R}=T_{i j k}^{f u l l} .
\end{gathered}
$$

Using these relations, one can formally show that the quenched Lagrangian is equal to the full Lagrangian under the restrictions of the flavor indices and the identifications of the parameters

$$
\alpha=2\left(\frac{1}{3} D+F\right), \beta=\left(-\frac{5}{3} D+F\right), \gamma=2(D-F)
$$

although there is no reason why the parameters of two theories should be related to each other. For better comparison with $\chi \mathrm{PT}$ result, we will re-express our results using these relations except $\gamma$.

The axial current from the quenched chiral Lagrangian is given by

$$
\begin{array}{r}
J_{\mu}^{a}=i \frac{f^{2}}{2} \operatorname{str}\left[T^{a}\left(\partial_{\mu} \Sigma^{\dagger} \Sigma-\partial_{\mu} \Sigma \Sigma^{\dagger}\right)\right]+v_{\mu}\left[\bar{B} \Omega_{-}^{A} B+2 \bar{B} B \Omega_{-}^{A}+3 \bar{T}^{\nu} \Omega_{-}^{A} T_{\nu}\right] \\
+2 \alpha \bar{B} S_{\mu} B \Omega_{+}^{A}+2 \beta \bar{B} S_{\mu} \Omega_{+}^{A} B+2 \gamma \bar{B} S_{\mu} B \operatorname{str}\left(\Omega_{+}^{A}\right) \\
+2 H \bar{T}^{\nu} S_{\mu} \Omega_{+}^{A} T_{\nu}+2 \gamma^{\prime} \bar{T}^{\nu} S_{\mu} T_{\nu} \operatorname{str}\left(\Omega_{+}^{A}\right)-\sqrt{\frac{3}{2}} C\left(\bar{T}^{\mu} \Omega_{+}^{A} B+\bar{B} \Omega_{+}^{A} T^{\mu}\right)
\end{array}
$$




$$
\begin{gathered}
\Omega_{ \pm}^{A}=\frac{1}{2}\left(\xi T^{A} \xi^{\dagger} \pm \xi^{\dagger} T^{A} \xi\right), \\
T^{A}=\frac{1}{2}\left[\begin{array}{cc}
\lambda^{A} & 0 \\
0 & \lambda^{A}
\end{array}\right],
\end{gathered}
$$

where $\lambda^{A}$ are Gell-Mann matrices. The numerical factors 2 and 3 in the second line is explained by the symmetry properties given in eq. (8).

The renormalization of the axial currents can be done by computing the diagrams given in the figures 1, 2 and 3 using Feynman rules derived from $\mathcal{L}_{\Phi}^{Q}$ and $\mathcal{L}_{B T \Phi}^{Q}$ and the vertices from eq. 18. All the diagrams in the figure 1 vanish: (a) vanishes since only closed quark loops are present (which cannot be present in the quenched approximation) and the others are zero due to the property $v \cdot S=0$. Then, the matrix element of $J^{A}$ for the octet baryons $B_{i}$ and $B_{j}$ can be written in the form

$$
\begin{gathered}
\left\langle B_{i}\left|J^{A}\right| B_{j}\right\rangle=\bar{u}_{B_{i}} \gamma^{\mu} \gamma^{5} u_{B_{j}} t_{i j}^{A}\left[1+\sum_{a \leq b}\left(\alpha_{i j, a b}^{A}-\lambda_{i j, a b}\right) X_{a b}+\sum_{a \leq b}\left(\beta_{i j, a b}^{A}-\rho_{i j, a b}\right) Y_{a b}\right], \\
X_{a b}=\frac{M_{a b}^{2}}{16 \pi^{2} f^{2}} \ln \frac{M_{a b}^{2}}{\mu^{2}}, \\
Y_{a b}=\frac{\left(\alpha_{\Phi} M_{a a}^{2}-m_{0}^{2}\right) X_{a a}-\left(\alpha_{\Phi} M_{b b}^{2}-m_{0}^{2}\right) X_{b b}}{3\left(M_{a a}^{2}-M_{b b}^{2}\right)}, \\
Y_{a a}=\frac{1}{48 \pi^{2} f^{2}}\left[\left(2 \alpha_{\Phi} M_{a a}^{2}-m_{0}^{2}\right) \ln \frac{M_{a a}^{2}}{\mu^{2}}+\left(\alpha_{\Phi} M_{a a}^{2}-m_{0}^{2}\right)\right],
\end{gathered}
$$

where $t_{i j}^{A}$ is the tree level result, $\lambda_{i j, a b}$ and $\rho_{i j, a b}$ are the wavefunction renormalization factors without and with the hairpin vertex, and $\alpha_{i j, a b}^{A}$ and $\beta_{i j, a b}^{A}$ are the one-loop corrections without and with the hairpin vertex. We list the coefficients at the end of this article. Here we use $m_{u}=m_{d}$.

The $\mu$ is the scale introduced in the dimensional regulazation. The scale dependence is cancelled by the counter terms obtained by the $O\left(m_{q}\right)$ Lagragians such as

$$
\frac{\left(\mu^{2}\right)^{4-d}}{\Lambda_{\chi}} \bar{B}\left(\xi^{\dagger} m \xi^{\dagger}+\xi m \xi\right) S^{\mu} A_{\mu} B
$$

where $m$ is the quark mass matrix. Including the counter terms, the function $X_{a b}$ changes to

$$
X_{a b} \rightarrow \frac{M_{a b}^{2}}{16 \pi f^{2}}\left[\ln \frac{M_{a b}^{2}}{\mu^{2}}+c(\mu)\right]
$$


where $c(\mu)$ is the finite part of the counter term. We take $\mu \simeq \Lambda_{\chi} \sim 1 \mathrm{GeV}$ and neglect the finite part. This is fully justified near the chiral limit since $\left|\ln \left(M_{a b}^{2} / \mu^{2}\right)\right| \gg c(\mu)$. In the real world the logarithmic correction is not significantly large but we expect it to give an estimate of the actual size of the corrections [10].

It is interesting to compare the full and the quenched chiral corrections to one of the above matrix elements. We choose the isovetor axial charge of the proton, $g_{A}$ for this. It is equal to the $\left\langle p\left|J^{1+i 2}\right| n\right\rangle$ due to the isospin symmetry. Quenched lattice simulation data for this quantity is also availiable [11]. Jenkins and Manohar computed full chiral logarithmic contributions to the baryon axial vector currents including both intermediate octet and decuplet states [8, 9]. Their full chiral result for $g_{A}$ is

$$
\begin{array}{r}
g_{A}=(D+F)\left[1-\left\{1+2(D+F)^{2}+\frac{2 C^{2}(9 D+9 F+25 H)}{81(D+F)}\right\} \frac{M_{\pi}^{2}}{16 \pi^{2} f^{2}} \ln \frac{M_{\pi}^{2}}{\mu^{2}}\right. \\
-\left\{\frac{1}{2}+\frac{15 D^{3}-D^{2} F+3 D F^{2}+33 F^{3}}{6(D+F)}+\frac{C^{2}(-27 D+45 F+20 H)}{162(D+F)}\right\} \frac{M_{K}^{2}}{16 \pi^{2} f^{2}} \ln \frac{M_{K}^{2}}{\mu^{2}} \\
\left.-\frac{(-D+3 F)^{2}}{3} \frac{M_{\eta}^{2}}{16 \pi^{2} f^{2}} \ln \frac{M_{\eta}^{2}}{\mu^{2}}\right] .
\end{array}
$$

On the other hand, our result for the quenched $g_{A}$ is

$$
\begin{array}{r}
g_{A}^{Q}=(D+F)\left[1+\left\{\frac{4(D-3 F)\left(D^{2}+2 D F+3 D \gamma+3 F \gamma\right)}{3(D+F)}\right.\right. \\
\left.+\frac{C^{2}(-15 D+9 F-10 H)}{27(D+F)}\right\} \frac{M_{\pi}^{2}}{16 \pi^{2} f^{2}} \ln \frac{M_{\pi}^{2}}{\mu^{2}} \\
\left.+2(-D+3 F)^{2} \frac{1}{48 \pi^{2} f^{2}}\left\{\left(2 \alpha_{\Phi} M_{\pi}^{2}-m_{0}^{2}\right) \ln \frac{M_{\pi}^{2}}{\mu^{2}}+\left(\alpha_{\Phi} M_{\pi}^{2}-m_{0}^{2}\right)\right\}\right] .
\end{array}
$$

In full theory, having only octet states, there are two free parameters $D$ and $F$ in their expressions. A $\chi^{2}$ fit to the hyperon semileptonic decays gives $D \sim 0.56$ and $F \sim 0.33$. Inclusion of the intermediate decuplet states needs two additional parameters $C^{2}$ and $H$. Jenkins and Manohar determined the parameter $C^{2}$ by fitting the $\Delta \rightarrow N \pi$ decay rate, $|C| \sim 1$.6. The three parameter fit yielded $D \sim 0.61, F \sim 0.40$ and $H \sim-1.9$. They used $\mu \sim 1 \mathrm{GeV}$.

The quenched chiral expressions for the axial currents given by eq.(21) need seven parameters $D, F, C^{2}, H, \gamma, \alpha_{\Phi}$ and $m_{0}$. One way to determine the values of the parameters 
is to fit to the quenched lattice data for the axial currents obtained on the physical quark masses. However, such simulation data is not yet available at present and we are interested in the qualitative comparison between the full chiral and the quenched chiral behaviors of the physical quantities rather than actual values of the parameters. Thus, for the sake of comparison, we use the values for the parameters $D, F, C^{2}$ and $H$ obtained by Jenkins and Manohar. We use the physical $\eta^{\prime}$ mass for the value of the parameter $m_{0}$. We determine the value of the parameter $\gamma$ by the relation $\gamma=2(D-F)$ and choose $\alpha_{\Phi}=0$. Our study shows that the variation of our result is less than $10 \%$ for the values $\gamma=0 \sim 1$ and the dependence on $\alpha_{\Phi}$ is much weaker (the variation less than $3 \%$ for $\alpha_{\Phi}=-1 \sim+1$ ).

In the figure, we show the full and the quenched chiral behaviors of the nucleon isovector axial charge with only octet states and both octet and decuplet states using the parameter values mentioned in the above (in the full chiral calculations, the $K-\pi$ and the $\eta-K$ mass differences are fixed at the physical values). In the quenched chiral calculations the meson loops with the $s$ flavor do not contribute. Full $\chi \mathrm{PT}$ result depends on pion mass visibly. However, in the region $4 m_{\pi}^{\text {physical }}$ and $6 m_{\pi}^{\text {physical }}$, the two plots of the $g_{A}^{Q}$ are almost flat with flatter behavior for octet states only. Of course these results are reliable only for sufficieltly small pion mass because the higher order corrections become important as the pion mass increases and we should take this figure as only an indication. There exists a quenched lattice simulation data of the axial charge [11] but due to the reason stated in the above, we can not compare our one loop result with this lattice data.

In short, one can see that in the limit $m_{\pi} \rightarrow 0$,

$$
g_{A} \rightarrow(D+F), g_{A}^{Q} \rightarrow c^{Q} \ln m_{\pi} .
$$

This singular behavior of the axial charge of the octet baryons, $g_{A}^{Q}$, is due to the quenched approximation to QCD. Similar to the case cosidered in observables of heavy-light meson system [12], the quenched artifact in the axial charge occurs as a leading singularity.

Finally we give the expressions for the coefficients appearing in eq. 21. The expressions 
for the $t_{i j}^{A}$ are

$$
\begin{array}{r}
t_{p p}^{8}=\frac{-D+3 F}{2 \sqrt{3}}, t_{p n}^{1+i 2}=D+F, t_{\Lambda \Sigma^{-}}^{1+i 2}=\sqrt{\frac{2}{3}} D, t_{\Xi^{0} \Xi^{-}}^{1+i 2}=D-F, \\
t_{p \Lambda}^{4+i 5}=-\frac{D+3 F}{\sqrt{6}}, t_{\Lambda \Xi^{-}}^{4+i 5}=\frac{-D+3 F}{\sqrt{6}}, t_{n \Sigma^{-}}^{4+i 5}=D-F, t_{\Sigma^{0} \Xi^{-}}^{4+i 5}=\frac{D+F}{\sqrt{2}}=\frac{1}{\sqrt{2}} t_{\Sigma^{+} \Xi^{0}}^{4+i 5} .
\end{array}
$$

The coefficients $\lambda_{i j, a b}$ and $\rho_{i j, a b}$ are defined by

$$
\begin{aligned}
\lambda_{i j, a b} & =\left(\lambda_{i, a b}+\lambda_{j, a b}\right) / 2, \\
\rho_{i j, a b} & =\left(\rho_{i, a b}+\rho_{j, a b}\right) / 2 .
\end{aligned}
$$

The diagrams (a) and (b) in the figure 2 yield

$$
\begin{gathered}
\lambda_{\Lambda, u u}=-\frac{1}{3} D^{2}+4 D F-3 F^{2}-(4 D-6 F) \gamma+\frac{1}{2} C^{2}, \\
\lambda_{\Lambda, u s}=-5 D^{2}+2 D F+3 F^{2}+\frac{1}{2} C^{2}, \quad \lambda_{\Lambda, s s}=(D+3 F) \gamma, \\
\lambda_{\Sigma, u u}=-D^{2}+3 F^{2}+6 F \gamma+\frac{1}{6} C^{2}, \\
\lambda_{\Sigma, u s}=-D^{2}+6 D F-3 F^{2}+\frac{5}{6} C^{2}, \quad \lambda_{\Sigma, s s}=3(-D+F) \gamma, \\
\lambda_{N, u u}=\lambda_{\Sigma, u u}+\lambda_{\Sigma, u s}+\lambda_{\Sigma, s s}, \quad \lambda_{N, u s}=\lambda_{N, s s}=0, \\
\lambda_{\Xi, u u}=\lambda_{\Sigma, s s}, \quad \lambda_{\Xi, u s}=\lambda_{\Sigma, u s}, \quad \lambda_{\Xi, s s}=\lambda_{\Sigma, u u} .
\end{gathered}
$$

The diagrams (c) and (d) in the figure 2 yield

$$
\begin{gathered}
\rho_{\Lambda, u u}=-\frac{3}{2}\left(\frac{4}{3} D-2 F\right)^{2}, \quad \rho_{\Lambda, u s}=3\left(\frac{4}{3} D-2 F\right)\left(\frac{1}{3} D+F\right), \quad \rho_{\Lambda, s s}=-\frac{3}{2}\left(\frac{1}{3} D+F\right)^{2} \gamma, \\
\rho_{\Sigma, u u}=-6 F^{2}-\frac{1}{3} C^{2}, \quad \rho_{\Sigma, u s}=6 F(D-F)+\frac{2}{3} C^{2}, \quad \rho_{\Sigma, s s}=-\frac{3}{2}(D-F)^{2}-\frac{1}{3} C^{2}, \\
\rho_{N, u u}=\rho_{\Sigma, u u}+\rho_{\Sigma, u s}+\rho_{\Sigma, s s}, \quad \rho_{N, u s}=\rho_{N, s s}=0,
\end{gathered}
$$




$$
\rho_{\Xi, u u}=\rho_{\Sigma, s s}, \quad \rho_{\Xi, u s}=\rho_{\Sigma, u s}, \quad \rho_{\Xi, s s}=\rho_{\Sigma, u u} .
$$

The $\alpha_{i j, a b}^{A}$ corresponds to the digrams without the hairpin in the figure 3. Our result for the $\alpha_{i j, a b}^{A}$ is

$$
\begin{aligned}
& \alpha_{p n, u u}^{1+i 2}=\frac{2 D(D-3 F)(-D+F)}{3(D+F)}+(D-3 F) \gamma+\frac{2 C^{2}(6 D+18 F-5 H)}{27(D+F)} \\
& \alpha_{p n, u s}^{1+i 2}=\alpha_{p n, s s}^{1+i 2}=0 \\
& \alpha_{\Lambda \Sigma^{-}, u u}^{1+i 2}=\frac{4 D(-D+3 F)}{9}+\frac{2(D-3 F) \gamma}{3}+\frac{C^{2}(-6 D+18 F-18 \gamma-5 H)}{54 D} \text {, } \\
& \alpha_{\Lambda \Sigma^{-}, u s}^{1+i 2}=\frac{D(D-3 F)}{9}+\frac{C^{2}(48 D+72 F-5 H)}{108 D}, \\
& \alpha_{\Lambda \Sigma^{-}, s s}^{1+i 2}=\frac{(D-3 F) \gamma}{3}+\frac{C^{2} \gamma}{3 D} \\
& \alpha_{\Xi^{0} \Xi^{-}, u u}^{1+i 2}=(D-F) \gamma+\frac{-4 C^{2} \gamma}{9(D-F)} \\
& \alpha_{\Xi^{0} \Xi^{-}, u s}^{1+i 2}=\frac{-D^{3}+3 D^{2} F-27 D F^{2}+9 F^{3}}{9(-D+F)}+\frac{C^{2}(18 D+18 F+5 H)}{81(D-F)}, \\
& \alpha_{\Xi^{0} \Xi^{-}, s s}^{1+i 2}=\frac{D^{3}+3 D^{2} F+9 D F^{2}-9 F^{3}}{9(-D+F)}-2 F \gamma+\frac{C^{2}(72 F+72 \gamma+5 H)}{162(D-F)}, \\
& \alpha_{p \Lambda, u u}^{4+i 5}=\frac{D^{3}+45 D^{2} F-81 D F^{2}+27 F^{3}}{18(D+3 F)}+\frac{(7 D-15 F) \gamma}{6}+\frac{C^{2}(9 D+9 F-5 H)}{9(D+3 F)}, \\
& \alpha_{p \Lambda, u s}^{4+i 5}=\frac{-25 D^{3}+63 D^{2} F-27 D F^{2}-27 F^{3}}{18(D+3 F)}+\frac{-\left(C^{2}(6 D-18 F+5 H)\right)}{18(D+3 F)}, \\
& \alpha_{p \Lambda, s s}^{4+i 5}=\frac{-((D+3 F) \gamma)}{6} \\
& \alpha_{\Lambda \Xi^{-}, u u}^{4+i 5}=\frac{(-D+F)\left(5 D^{2}-6 D F+9 F^{2}\right)}{6(-D+3 F)}+\frac{(7 D-9 F) \gamma}{6}+\frac{C^{2}(-9 D+9 F-18 \gamma-5 H)}{27(-D+3 F)} \\
& \alpha_{\Lambda \Xi^{-}, \text {us }}^{4+i 5}=\frac{4 D\left(D^{2}+6 D F-9 F^{2}\right)}{9(-D+3 F)}+\frac{2 C^{2}(3 D-9 F-5 H)}{27(-D+3 F)}, \\
& \alpha_{\Lambda \Xi^{-}, s s}^{4+i 5}=\frac{(D+3 F)\left(-5 D^{2}+6 D F-9 F^{2}\right)}{18(-D+3 F)}-\frac{(D+9 F) \gamma}{6}+\frac{C^{2}(D+3 F+6 \gamma)}{9(-D+3 F)},
\end{aligned}
$$




$$
\begin{aligned}
& \alpha_{n \Sigma^{-}, u u}^{4+i 5}=\frac{D^{3}+9 D^{2} F-9 D F^{2}-9 F^{3}}{18(-D+F)}+\frac{(D-5 F) \gamma}{2}+\frac{C^{2}(9 D+45 F+18 \gamma+5 H)}{81(D-F)}, \\
& \alpha_{n \Sigma^{-}, u s}^{4+i 5}=\frac{-D^{3}+3 D^{2} F-27 D F^{2}+9 F^{3}}{18(-D+F)}+\frac{C^{2}(18 D+18 F+5 H)}{162(D-F)}, \\
& \alpha_{n \Sigma^{-}, s s}^{4+i 5}=\frac{(D-F) \gamma}{2}+\frac{-2 C^{2} \gamma}{9(D-F)}, \\
& \alpha_{\Sigma^{0} \Xi^{-}, u u}^{4+i 5}=\frac{(D-F)\left(-D^{2}+6 D F+3 F^{2}\right)}{6(D+F)}+\frac{(D-3 F) \gamma}{2}+\frac{C^{2}(-9 D+9 F-5 H)}{81(D+F)} \\
& \alpha_{\Sigma^{0} \Xi^{-}, u s}^{4+i 5}=\frac{(-D+F)\left(D^{2}+3 F^{2}\right)}{3(D+F)}+\frac{2 C^{2}(27 D+45 F-10 H)}{81(D+F)}, \\
& \alpha_{\Sigma^{0} \Xi^{-}, s s}^{4+i 5}=\alpha_{\Sigma^{0} \Xi^{-}, u u}^{4+i 5}, \quad \alpha_{\Sigma^{+} \Xi^{0}, a b}^{4+i 5}=\alpha_{\Sigma^{0} \Xi^{-}, a b}^{4+i 5}, \\
& 3 \\
& \alpha_{p p, u u}^{8}=\frac{2 D(D-3 F)}{3}+(D-3 F) \gamma+\frac{5 C^{2} H}{9(D-3 F)}, \alpha_{p p, u s}^{8}=\alpha_{p p, s s}^{8}=0 .
\end{aligned}
$$

The diagrams with the hairpin in the figure 3 yield the results for $\beta_{i j, a b}^{A}$ which are

$$
\begin{gathered}
\beta_{p n, u u}^{1+i 2}=\frac{(-D+3 F)^{2}}{2}, \beta_{p n, u s}^{1+i 2}=\beta_{p n, s s}^{1+i 2}=0 \\
\beta_{\Lambda \Sigma^{-}, u u}^{1+i 2}=\frac{2 F(-2 D+3 F)}{3}+\frac{2 C^{2}(-2 D+3 F)}{9 D} \\
\beta_{\Lambda \Sigma^{-}, u s}^{1+i 2}=\frac{2\left(D^{2}-2 D F+3 F^{2}\right)}{3}+\frac{C^{2}(5 D-3 F)}{9 D} \\
\beta_{\Lambda \Sigma^{-}, s s}^{1+i 2}=\frac{-D^{2}}{6}-\frac{D F}{3}+\frac{F^{2}}{2}+\frac{-\left(C^{2}(D+3 F)\right)}{9 D} \\
\beta_{\Xi^{0} \Xi^{-}, u u}^{1+i 2}=\frac{(D-F)^{2}}{2}+\frac{C^{2}(-36 D+36 F-5 H)}{81(D-F)} \\
\beta_{\Xi^{0} \Xi^{-}, u s}^{1+i 2}=-2(D-F) F+\frac{2 C^{2}(18 D+18 F+5 H)}{81(D-F)} \\
\beta_{\Xi^{0} \Xi^{-}, s s}^{1+i 2}=2 F^{2}+\frac{-\left(C^{2}(72 F+5 H)\right)}{81(D-F)}
\end{gathered}
$$




$$
\begin{aligned}
& \beta_{p \Lambda, u u}^{4+i 5}=\frac{1}{4}+\frac{2 D^{2}}{3}-3 D F+3 F^{2} \\
& \beta_{p \Lambda, u s}^{4+i 5}=-\frac{1}{2}+\frac{-D^{2}}{6}+\frac{3 F^{2}}{2} \\
& \beta_{p \Lambda, s s}^{4+i 5}=\frac{1}{4} \\
& \beta_{\Lambda \Xi^{-}, u u}^{4+i 5}=\frac{1}{4}+\frac{2 D^{2}-5 D F+3 F^{2}}{3}+\frac{4 C^{2}(-2 D+3 F)}{9(-D+3 F)}, \\
& \beta_{\Lambda \Xi^{-}, u s}^{4+i 5}=-\frac{1}{2}+\frac{-D^{2}-10 D F+15 F^{2}}{6}+\frac{2 C^{2}(5 D-3 F)}{9(-D+3 F)} \\
& \beta_{\Lambda \Xi^{-}, s s}^{4+i 5}=\frac{1}{4}+\frac{F(D+3 F)}{3}+\frac{2 C^{2}(D+3 F)}{9(D-3 F)}, \\
& \beta_{n \Sigma^{-}, u u}^{4+i 5}=\frac{1}{4}+F(-D+3 F)+\frac{2 C^{2}(D-3 F)}{9(D-F)}, \\
& \beta_{n \Sigma^{-}, u s}^{4+i 5}=-\frac{1}{2}+\frac{D^{2}-4 D F+3 F^{2}}{2}+\frac{2 C^{2}(D-3 F)}{9(-D+F)}, \\
& \beta_{n \Sigma^{-}, s s}^{4+i 5}=\frac{1}{4} \\
& \beta_{\Sigma^{0} \Xi^{-}, \text {uu }}^{4+i 5}=\frac{1}{4}+F(-D+F)+\frac{2 C^{2}(-9 D-9 F+5 H)}{81(D+F)}, \\
& \beta_{\Sigma^{0} \Xi^{-}, u s}^{4+i 5}=-\frac{1}{2}+\frac{D^{2}-2 D F+5 F^{2}}{2}+\frac{4 C^{2}(9 D+9 F-5 H)}{81(D+F)}, \\
& \beta_{\Sigma^{0} \Xi^{-}, s s}^{4+i 5}=\frac{1}{4}+F(-D+F)+\frac{2 C^{2}(-9 D-9 F+5 H)}{81(D+F)} \\
& \beta_{\Sigma^{+} \Xi^{0}, a b}^{4+i 5}=\beta_{\Sigma^{0} \Xi^{-}, a b}^{4+i 5} \\
& \beta_{p p, u u}^{8}=\frac{(-D+3 F)^{2}}{2}, \beta_{p p, u s}^{8}=\beta_{p p, s s}^{8}=0 .
\end{aligned}
$$

\section{Acknowledgements}

We are thankful to T.-S. Park and P. Ko for useful discussions. This work was supported by the Korean Science and Engineering Foundation through the Center for Theoretical Physics. 


\section{References}

[1] See for example, Nucl. Phys. B 47 (Proc. Suppl.), (1996).

[2] S.Sharpe, Phys. Rev. D 41, 3233 (1990).

[3] C.Bernard and M.F.L.Golterman, Nucl. Phys. B 17, 217 (1993).

[4] J.N.Labrenz and S.Sharpe, U. of W preprint PP \#96-07, hep-lat/9605034.

[5] S.Kim and D.K.Sinclair, Phy. Rev. D 52 R2614 (1995).

[6] S.Sharpe, in preparation

[7] H.Georgi, Phys. Lett., B 240447 (1990).

[8] E.Jenkins and A.V.Manohar, Phys. Lett., B 255558 (1991).

[9] E.Jenkins and A.V.Manohar, Phys. Lett., B 259353 (1991).

[10] M.A.Luty and M.White, Phys. Lett., B 319261 (1993).

[11] K.F.Liu, S.J.Dong, T.Draper, and J.M.Wu, Phys. Rev.,D 494755 (1994).

[12] S.R.Sharpe and Y.Zhang, Phys. Rev.,D 535125 (1996). 


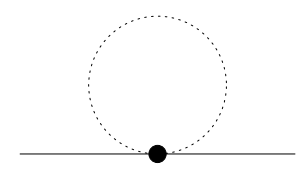

(a)

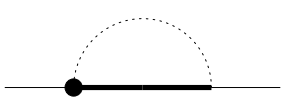

(d)

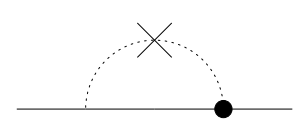

( g)

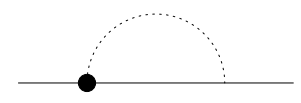

(b)

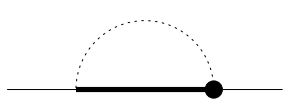

(e)

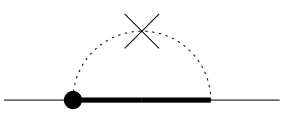

( h )

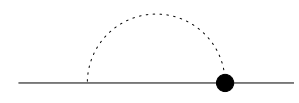

(c)

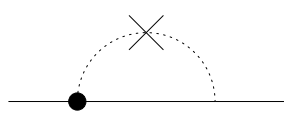

(f)

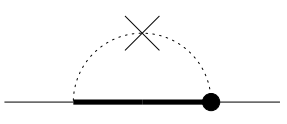

(i)

Figure 1: Vanishing graphs. The solid dots represent the vertices steming from the axial current.

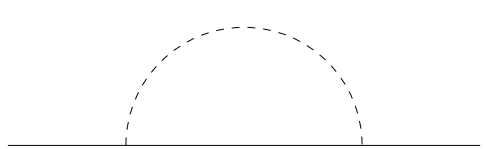

( a )

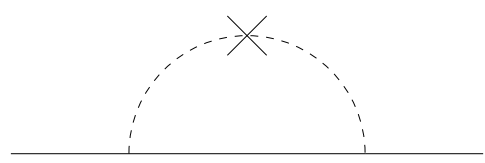

(c)

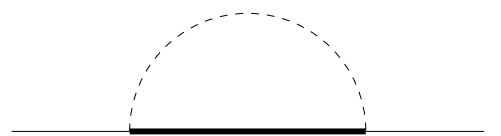

( b )

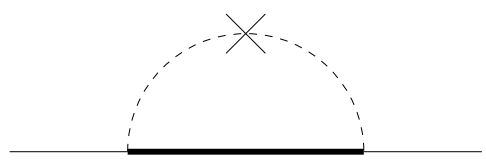

( d )

Figure 2: Wavefunction renormalization graphs. The hairpin represents the vertex $i\left(\alpha_{\Phi} p^{2}-\right.$ $\left.m_{0}^{2}\right) / 3$. 


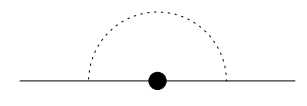

(a)

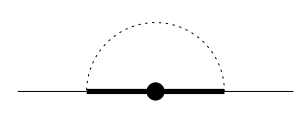

(d)

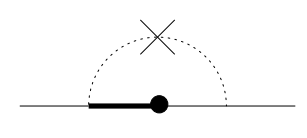

(g)

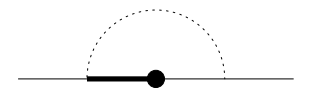

(b)

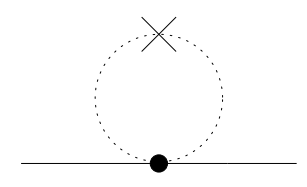

(e)

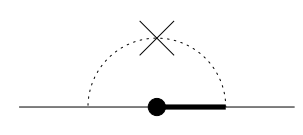

(h)

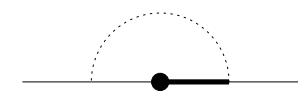

(c)

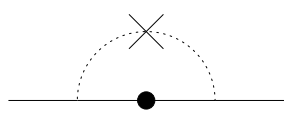

(f)

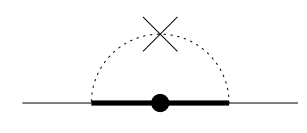

(i)

Figure 3: Graphs contributing to the coefficients $\alpha_{i j, a b}^{A}$ and $\beta_{i j, a b}^{A}$. The solid dots represent the vertices steming from the axial current. The hairpin represents the vertex $i\left(\alpha_{\Phi} p^{2}-m_{0}^{2}\right) / 3$.

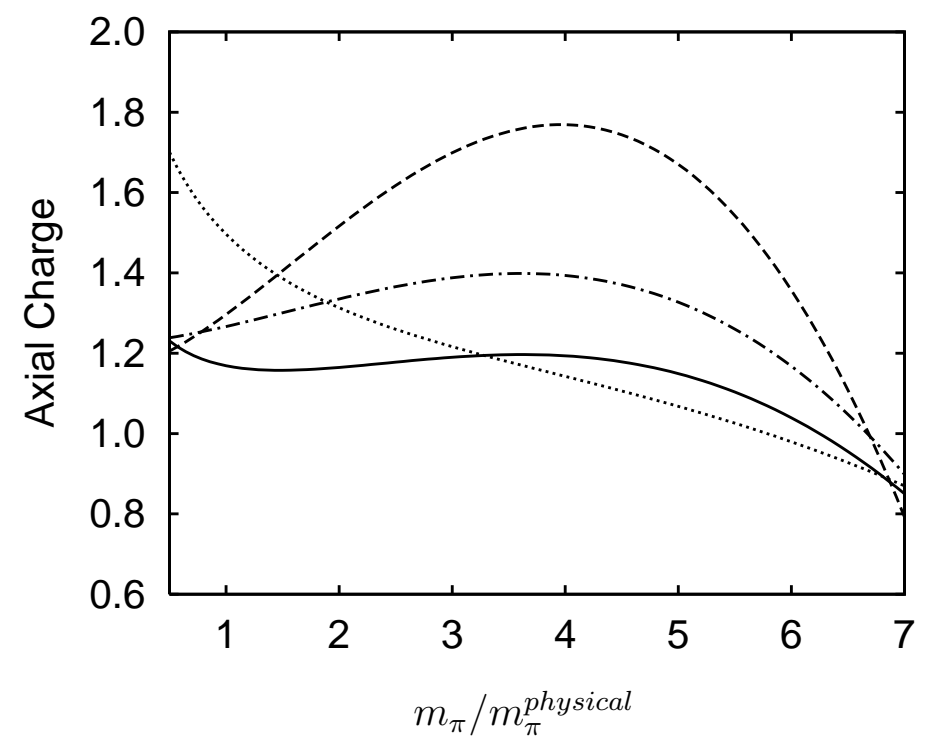

Figure 4: Chiral behaviors of the nucleon isovector axial charge from $\mathrm{Q} \chi \mathrm{PT}$ and $\chi \mathrm{PT}$ with only octet intermediate states and both octet and decuplet states. The solid, dotted, dashed, and dot-dashed lines show the chiral behaviors form $\mathrm{Q} \chi \mathrm{PT}$ with octets, $\mathrm{Q} \chi \mathrm{PT}$ with both octets and decuplets, $\chi \mathrm{PT}$ with octets, and $\chi \mathrm{PT}$ with both octets and decuplets, in order. 